\section{Tracheal tube kinking}

To the Editor:

We would like to report an unusual cause of kinking of a PVC tracheal tube which occurred during nasal intubation.

A 24-yr-old man, ASA class I, was scheduled for intraoral surgery to excise a swelling of the lower jaw. His preoperative airway examination was unremarkable. After premedication with pentazocine and promethazine, anaesthesia was induced with glycopyrrolate, thiopentone followed by succinylcholine $100 \mathrm{mg}$. Larnyngoscopy was performed and an $8 \mathrm{~mm}$ PVC cuffed tracheal tube was inserted through the right nostril. The tip of the tube was seen to enter the glottis when the laryngoscope was withdrawn and the tube was pushed in further without undue resistance. The cuff was inflated and the tube connected to the breathing circuit but the reservoir bag could not be deflated on squeezing. Instead, it kept inflating until the pressure limiting valve on the circuit opened. On repeat laryngoscopy, the tip of the tube was seen end-on with the inflated cuff pushing the epiglottis forward. The tube was taken out immediately and the trachea was re-intubated with another tube. Anaesthesia subsequently was uneventful.

Closer examination of the tube on removal showed a residual kink in the intracuff portion. On cutting open the cuff, a large transverse nick $(6.3 \times 3.4 \mathrm{~mm})$ was seen at the site of the residual kink. Examination of 35 randomly chosen tubes of the same size revealed that the much smaller nicks $(4.3 \pm 0.5 \times 3.02 \pm 0.5 \mathrm{~mm})$ which are probably made during manufacture, across the path of the inflating lumen to open into the cuff. We presume that kinking in our case occurred because the larger nick formed a weak point.

We suggest that the possibility of kinking in the region of nick should be kept in mind while inserting the nasal tube.

Bajjit Singh MD

K.K. Gombar MD

Balbir Chhabra DA MS

Department of Anaesthesia

Medical College and Hospital

Rohtak 124001

India

\section{Oral midazolam and post- operative behaviour in children}

To the Editor:

Children who resist or cry during anaesthetic induction may exhibit adverse behaviour changes postoperatively. ${ }^{1}$ We conducted a small prospective double-blind, randomized study examining the impact of oral midazolam premedication on children's behaviour during the postoperative period. We sought to test the hypothesis that preoperative sedation might mitigate against adverse postoperative behaviour.

This study was approved by the institutional review board of our hospital. All subjects were outpatients and without history of behavioural problems. Following enrollment, subjects were given either oral midazolam 0.5 $\mathrm{mg} \cdot \mathrm{kg}^{-1}$ (up to a maximum of $10 \mathrm{mg}$ ) or an equal volume of bitter tasting placebo. Approximately 15 to $20 \mathrm{~min}$ after ingestion of premedication, the child was anaesthetized in a parent's presence with halothane and nitrous oxide in oxygen via mask. Upon enrollment and at anaesthetic induction subjects were assessed for sedation using a visual analogue scale. Telephone follow-up was conducted at one and four weeks following surgery.

There were 22 children studied in the placebo group and 22 in the midazolam group. The mean age in the placebo group was $2.7 \mathrm{yr}$ (range 1.1 to $6 \mathrm{yr}$ ). The mean age in the midazolam group was $3.6 \mathrm{yr}$ (range 1.2 to $10 \mathrm{yr}$ ) (NS). The baseline evaluation scores for the two groups were not different. The midazolam group were more sedated at induction than were the placebo group $(P<0.01)$. Four of the 22 placebo subjects $(18 \%)$ ex perienced adverse behavioural sequelae during the first postoperative week; 11 of the 22 midazolam subjects (50\%) experienced adverse behavioural sequelae during this period $(P=0.056)$. Types of behaviour changes included nightmares, fearfulness, and food rejection. In both placebo and midazolam groups, the behaviour changes were resolved within 14 days following surgery in all children.

Our findings regarding postoperative behaviour were unexpected. The midazolam appeared to be associated with an increased incidence of adverse postoperative behaviour. This observation approached but did not achieve statistical significance using a two-tailed Fisher exact test. However, our population was too small and our evaluations too imprecise to conclude that oral midazolam premedication worsens postoperative behaviour. Larger studies performed in various anaesthetic settings are needed to explore this issue. 